\title{
Applications of indocyanine green in brain tumor surgery: review of clinical evidence and emerging technologies
}

\author{
${ }^{*}$ Clare W. Teng, BA, ${ }^{1,2}$ Vincent Huang, BA, ${ }^{1,2}$ Gabriel R. Arguelles, BA, ${ }^{1,2}$ Cecilia Zhou, BS, ${ }^{1,2}$ \\ Steve S. Cho, MD, MTR, ${ }^{1}$ Stefan Harmsen, PhD, ${ }^{1}$ and John Y. K. Lee, MD, MSCE${ }^{1}$ \\ 1Department of Neurosurgery, Hospital of the University of Pennsylvania, Philadelphia; and 2Perelman School of Medicine, \\ University of Pennsylvania, Philadelphia, Pennsylvania
}

\begin{abstract}
Indocyanine green (ICG) is a water-soluble dye that was approved by the FDA for biomedical purposes in 1956. Initially used to measure cardiocirculatory and hepatic functions, ICG's fluorescent properties in the near-infrared (NIR) spectrum soon led to its application in ophthalmic angiography. In the early 2000s, ICG was formally introduced in neurosurgery as an angiographic tool. In 2016, the authors' group pioneered a novel technique with ICG named second-window ICG (SWIG), which involves infusion of a high dose of ICG $(5.0 \mathrm{mg} / \mathrm{kg})$ in patients 24 hours prior to surgery. To date, applications of SWIG have been reported in patients with high-grade gliomas, meningiomas, brain metastases, pituitary adenomas, craniopharyngiomas, chordomas, and pinealomas.

The applications of ICG have clearly expanded rapidly across different specialties since its initial development. As an NIR fluorophore, ICG has advantages over other FDA-approved fluorophores, all of which are currently in the visible-light spectrum, because of NIR fluorescence's increased tissue penetration and decreased autofluorescence. Recently, interest in the latest applications of ICG in brain tumor surgery has grown beyond its role as an NIR fluorophore, extending into shortwave infrared imaging and integration into nanotechnology. This review aims to summarize reported clinical studies on ICG fluorescence-guided surgery of intracranial tumors, as well as to provide an overview of the literature on emerging technologies related to the utility of ICG in neuro-oncological surgeries, including the following aspects: 1) ICG fluorescence in the NIR-II window; 2) ICG for photoacoustic imaging; and 3) ICG nanoparticles for combined diagnostic imaging and therapy (theranostic) applications.
\end{abstract}

https://thejns.org/doi/abs/10.3171/2020.10.FOCUS20782

KEYWORDS indocyanine green; fluorescence-guided surgery; brain tumor; NIR-II window; photoacoustic imaging; theranostics

$\mathrm{I}$ NDOCYANINE green (ICG), a tricarbocyanine, is a watersoluble dye originally developed by Kodak Research Laboratories for near-infrared (NIR) photography in 1955. Biomedical applications of ICG began in 1956, when it received FDA approval for diagnostic use in determining cardiocirculatory and hepatic functions. ${ }^{1}$ Whereas early studies focused on tracking ICG levels in the serum, discovery of ICG's fluorescent properties in the 1970s expanded its use to ophthalmic angiography. This development truly revealed the potential of ICG to provide visual guidance in anatomical structures during surgical procedures, which built a foundation for image-guided surgery. ${ }^{2}$ In 2003, ICG was formally introduced in neurosurgery as an angiographic agent with a landmark study by Raabe et al., and it has since become a popular tool in vascular neurosurgery for intraoperative evaluation of aneurysms, arteriovenous malformations, and cortical perfusion ${ }^{3}$ (for the ICG timeline see Fig. 1).

The use of ICG in brain tumor surgery, in contrast, has remained at modest levels in clinical practice over the years. As early as 1996, Haglund et al. had described enhanced optical imaging by using ICG in 9 patients undergoing resection of high-grade gliomas (HGGs). ${ }^{4}$ Fluorescence-guided surgery (FGS) of brain tumors only began to accrue more interest in the early 2000 s, culminating in a phase III trial by Stummer et al. demonstrating improved progression-free survival in patients with malignant glioma treated with 5-aminolevulinic acid (5-ALA)

ABBREVIATIONS 5-ALA = 5-aminolevulinic acid; BBB = blood-brain barrier; DOX = doxorubicin; EPR = enhanced permeability and retention; FGS = fluorescence-guided surgery; GBM = glioblastoma; GTR = gross-total resection; HGG = high-grade glioma; ICG = indocyanine green; NIR = near infrared; PAI = photoacoustic imaging; PDT = photodynamic therapy; PTT = photothermal therapy; SBR = signal-to-background ratio; SPIO = superparamagnetic iron oxide; SPION = SPIO nanoparticle; SWIG = second-window ICG; SWIR = shortwave infrared.

SUBMITTED September 1, 2020. ACCEPTED October 23, 2020.

INCLUDE WHEN CITING DOI: 10.3171/2020.10.FOCUS20782.

* V.H. and G.R.A. contributed equally to this work. 


\begin{tabular}{|c|c|c|c|}
\hline \multirow[t]{2}{*}{$\begin{array}{l}1956 \\
\text { FDA approval of ICG for } \\
\text { cardiovascular and } \\
\text { hepatic indications }\end{array}$} & $\begin{array}{l}2003 \\
\text { Raabe et } \text { al. }^{3} \text { - ICG } \\
\text { angiogram introduced to } \\
\text { clinical neurosurgery }\end{array}$ & \multicolumn{2}{|c|}{$\begin{array}{l}2016 \\
\text { Lee et al.' - first clinical study } \\
\text { for Second Window ICG in } \\
\text { high-grade glioma }\end{array}$} \\
\hline & $\begin{array}{l}1996 \\
\text { Haglund et } \text { al. }^{4} \text { described } \\
\text { enhanced optical imaging } \\
\text { using ICG for brain tumor }\end{array}$ & $\begin{array}{c}2012 \\
\text { Madajewski et al. }{ }^{6} \text { introduce } \\
\text { high-dose ICG delayed } \\
\text { imaging in animal study }\end{array}$ & $\begin{array}{l}2018 \\
\text { Carr et al. } \text {. }^{35} \text { : discovery } \\
\text { of ICG off-peak } \\
\text { NIR-II emission }\end{array}$ \\
\hline
\end{tabular}

FIG. 1. Milestones of ICG for brain tumor surgery.

FGS. ${ }^{5}$ This led to a revival of interest in ICG as an optical imaging agent. A breakthrough took place in 2012, when Madajewski et al. proposed that administration of high-dose ICG $(7.5 \mathrm{mg} / \mathrm{kg}) 24$ hours before surgery allowed residual neoplasm detection in murine flank tumors. ${ }^{6}$ From this principle, our group pioneered a novel technique named second-window ICG (SWIG), in which high-dose ICG $(5 \mathrm{mg} / \mathrm{kg})$ is administered 24 hours prior to intraoperative imaging. In 2016, we conducted the firstin-human study, which demonstrated significant NIR contrast in Gd-enhancing gliomas in the operating room. ${ }^{7}$ To date, applications of SWIG have been reported in HGGs, meningiomas, brain metastases, pituitary adenomas, craniopharyngiomas, chordomas, and pinealomas. ${ }^{8-10}$

ICG remains the first and only FDA-approved NIR fluorophore. After intravenous administration, this small, amphiphilic molecule rapidly binds to albumin and other plasma proteins. ${ }^{11}$ ICG is almost exclusively metabolized by the liver and excreted into bile, with a mean initial decay rate of $18.5 \%$ per minute in healthy individuals. ${ }^{12}$ ICG fluorescence is excited by 778- to 806-nm light, with a peak emission of $835 \mathrm{~nm}$ in biological tissues. NIR fluorescent emission of ICG can penetrate tissue up to $15 \mathrm{~mm}$, compared with $3 \mathrm{~mm}$ for visible-light fluorophores. ${ }^{13} \mathrm{Be}-$ sides, ICG suffers from less tissue autofluorescence because it operates in the NIR spectrum, in which biological tissue mostly does not demonstrate fluorescence.

The tumor-targeting mechanism behind SWIG is known as the enhanced permeability and retention (EPR) effect, which stipulates that vascular permeability around solid tumors is pathologically enhanced due to structural breakdown, impaired lymphatic drainage, and increased permeability mediators. ${ }^{14}$

Lately, the application of ICG in brain tumor surgery has been extended beyond its role as simply an NIR fluorophore. This turning point has been fueled by two key developments: the investigation of the shortwave infrared (SWIR) window and the rapid growth of nanotechnology. In this review, we provide an overview of the clinical studies on ICG FGS of intracranial tumors conducted by our group and others (Table 1). We then summarize the literature on emerging technologies and their implications for the potential utility of ICG in neuro-oncological procedures.

\section{Clinical Trials of ICG for Fluorescence- Guided Intracranial Tumor Surgery}

ICG visualization can be accomplished without disrupting the surgeon's usual workflow under white-light illumination, due to existing capability in real-time overlay with both visible light and NIR fluorescence ${ }^{15}$ (Fig. 2). SWIG was first used to visualize gliomas in 2016. This study and subsequent reports in skull base tumors found that $\mathrm{Gd}$ enhancement on T1-weighted MRI was the strongest predictor of positive fluorescence, whereas nonenhancing tumors did not fluoresce, providing insight into the mechanism of ICG localization. ${ }^{7,16}$ In 2012, Ergin et al. had proposed using intravenous ICG injections to optically monitor blood-brain barrier (BBB) disruption, taking advantage of the same EPR effect proposed to underpin ICG accumulation in the tumor microenvironment. ${ }^{17}$ In the initial SWIG study from 2016, findings in patients with contrast-enhancing gliomas (12/15) were used to calculate diagnostic test characteristics. Using tumor pathology as the gold standard, SWIG demonstrated higher sensitivity and negative predictive value but lower specificity and a positive predictive value compared with visualization under white light; these test characteristics were later corroborated in intracranial metastases, meningiomas, and pituitary adenomas. ${ }^{18-20}$ Enhanced sensitivity and a negative predictive value may give neurosurgeons more confidence in detecting remaining neoplastic tissue and thus facilitating gross-total resection (GTR).

Establishing the novel clinical utility of ICG set the stage for studies in other brain tumor types. In 2018, ICG was used to successfully localize tumors displaying a high signal-to-background ratio (SBR) in $14 / 18$ patients with meningioma. ${ }^{19}$ In the remaining minority of patients, the surrounding brain parenchyma displayed a higher NIR signal than the tumor, an interesting phenomenon currently only seen in meningiomas. We hypothesized that this phenomenon of signal inversion may be due to autoquenching of ICG that can occur at high concentrations. ${ }^{21}$ At approximately the same time, ICG was demonstrated to localize intraparenchymal metastases in 13 patients with primary pathologies that included melanoma, lung, breast, colon, ovarian, and renal cancers. ${ }^{18}$

Of note, melanoma metastases had the lowest mean SBR at 3.65, which is attributed to melanin's absorption of light, compared with a mean SBR of 7.2 for other metastases. This observation was also corroborated by updated data in progress for publication. Other studies have demonstrated ICG's ability to visualize chordoma, craniopharyngioma, pineocytoma, and intraventricular tumors..$^{6,22,23}$

Because the ultimate goal of FGS is to maximize extent of resection, studies by Cho et al. in $2020^{24}$ and Teng et al. ${ }^{76}$ comparing NIR fluorescence to the gold standard postoperative MRI showed that the absence of re- 
TABLE 1. Summary of clinical studies of ICG application in brain tumors

\begin{tabular}{|c|c|c|}
\hline Authors \& Year & Tumor or Structure & Key Findings \\
\hline Lee et al., $2016^{7}$ & Glioma & $\begin{array}{l}\text { Mean SBR of } 9.5 \mathrm{w} / 5.0 \mathrm{mg} / \mathrm{kg} \text { SWIG in } 12 \mathrm{pts} \text { w/ Gd-enhancing tumors, deepest tumor visualized was } \\
13 \mathrm{~mm} \text { from dural surface, Gd enhancement on MRI correlates to SBR }(p=0.03)\end{array}$ \\
\hline $\begin{array}{l}\text { Ferroli et al., } \\
2011^{8}\end{array}$ & Glioma & $\begin{array}{l}25 \text { mg ICG video angiography in } 54 \text { HGGs, } 17 \text { LGGs, } 14 \text { meningiomas, } 12 \text { metastases, \& } 3 \text { hemangio- } \\
\text { blastomas can monitor blood flow in exposed tumoral \& peritumoral vessels }\end{array}$ \\
\hline Zeh et al., $2017^{9}$ & HGG & Mean SBR of $7.5 \mathrm{w} / 2.5-5.0 \mathrm{mg} / \mathrm{kg}$ SWIG in $10 \mathrm{pts}$, plateau of ICG NIRF up to $48 \mathrm{hrs}$ postinfusion \\
\hline Cho et al., $2020^{24}$ & $\begin{array}{l}\text { Newly diagnosed } \\
\text { HGG }\end{array}$ & $\begin{array}{l}\text { Mean SBR of } 6.8 \mathrm{w} / 2.5-5.0 \mathrm{mg} / \mathrm{kg} \text { SWIG in } 36 \mathrm{pts}, 90 \%(27 / 30) \text { of tumors } \leq 15 \mathrm{~mm} \text { from dural surface } \\
\text { were visualized, clean final NIR view correlated to GTR on postop MRI }(p<0.0001)\end{array}$ \\
\hline Lee et al., $2018^{19}$ & Meningioma & $\begin{array}{l}\text { Mean SBR of } 5.6 \mathrm{w} / 5.0 \mathrm{mg} / \mathrm{kg} \text { SWIG in } 14 / 18 \mathrm{pts} ; 4 / 18 \mathrm{pts} \text { demonstrated "inversion" in which immediate } \\
\text { parenchyma had higher SBR than the tumor }\end{array}$ \\
\hline Kim et al., $2019^{25}$ & Meningioma & $\begin{array}{l}12.5 \mathrm{mg} \text { ICG video angiography in } 42 \text { pts allows real-time monitoring of peritumoral blood flow, detection } \\
\text { of collateral venous circulation, \& flow restoration }\end{array}$ \\
\hline Lee et al., $20177^{18}$ & Metastases & $\begin{array}{l}\text { Mean SBR of } 6.62 \mathrm{w} / 5.0 \mathrm{mg} / \mathrm{kg} \text { SWIG in } 13 \mathrm{pts} \text {, melanoma metastases had lower SBR signal, deepest } \\
\text { tumor visualized was } 7 \mathrm{~mm} \text { from dural surface }\end{array}$ \\
\hline $\begin{array}{l}\text { Teng et al., in } \\
\text { press }^{76}\end{array}$ & Metastases & $\begin{array}{l}\text { Mean SBR of } 4.9 \mathrm{w} / 2.5-5.0 \mathrm{mg} / \mathrm{kg} \text { SWIG in } 47 \mathrm{pts} \text {, melanoma metastases had lower SBR signal, clean } \\
\text { final NIR view correlated to GTR on postop MRI }(p=0.007)\end{array}$ \\
\hline Jeon et al., $2019^{16}$ & Skull base tumors & $\begin{array}{l}\text { Mean SBR of } 3.9 \mathrm{w} / 5.0 \mathrm{mg} / \mathrm{kg} \text { SWIG in } 8 \text { pituitary adenomas, mean SBR of } 4.1 \text { in } 3 \text { craniopharyngio- } \\
\text { mas, mean SBR of } 2.1 \text { in } 4 \text { chordomas, Gd enhancement on MRI correlates to SBR ( } p=0.0003)\end{array}$ \\
\hline Hide et al., $2015^{26}$ & Skull base tumors & $\begin{array}{l}12.5 \text { mg ICG video angiography in } 26 \text { pituitary adenomas, } 4 \text { tuberculum sellae meningiomas, } 3 \text { cranio- } \\
\text { pharyngiomas, } 2 \text { chordomas, } 1 \text { Rathke cleft cyst, } 1 \text { dermoid cyst, \& } 1 \text { fibrous dysplasia strongly labels } \\
\text { landmark blood vessels in real time }\end{array}$ \\
\hline $\begin{array}{l}\text { Amano et al., } \\
2019^{27}\end{array}$ & Skull base tumors & $\begin{array}{l}\text { 6.25-12.5 mg ICG video angiography in } 15 \text { pituitary adenomas, } 3 \text { Rathke cleft cysts, } 1 \text { meningioma, \& } 1 \\
\text { pituicytoma can differentiate tumor margin \& define local vessel structure }\end{array}$ \\
\hline $\begin{array}{l}\text { Sandow et al., } \\
2015^{10}\end{array}$ & Skull base tumors & $\begin{array}{l}25 \text { mg ICG video angiography in } 22 \text { pituitary adenomas can either directly visualize tumor fluorescence } \\
\text { or indirectly detect tumor w/ higher background fluorescence }\end{array}$ \\
\hline Cho et al., $2020^{22}$ & Pineocytoma & SBR of $2.9 \mathrm{w} / 5.0 \mathrm{mg} / \mathrm{kg}$ SWIG in $1 \mathrm{pt}$ \\
\hline Cho et al., $2020^{31}$ & Pituitary stalk & $\begin{array}{l}2.5 \mathrm{mg} / \mathrm{kg} \text { SWIG allows for visualization of normal pituitary stalk despite distorted anatomy in } 1 \mathrm{pt} w / \\
\text { pituitary macroadenoma }\end{array}$ \\
\hline Hitti \& Lee, $2018^{23}$ & Intraventricular tumor & SWIG localizes to both normal choroid plexus \& choroid plexus papilloma for endoscopic resection in 1 pt \\
\hline Hojo et al., $2014^{28}$ & Hemangioblastoma & $\begin{array}{l}\text { 8-12.5 mg ICG video angiography in } 12 \text { pts facilitates preresection visualization of tumor \& feeder arter- } \\
\text { ies, \& postresection confirmation of total resection \& normal blood flow }\end{array}$ \\
\hline
\end{tabular}

HGG = high-grade glioma; $L G G$ = low-grade glioma; NIRF = near-infrared fluorescence; pt = patient.

sidual NIR signal postresection closely correlated to GTR of HGGs ( $\mathrm{p}<0.0001)$ and intracranial metastases $(\mathrm{p}=$ 0.007 ) on MRI. One limitation of these findings, however, is intrinsic to the SWIG technique itself. Although ICG is FDA approved, its current dosage of $2.5-5.0 \mathrm{mg} / \mathrm{kg}$ in SWIG clinical trials exceeds the $2.0 \mathrm{mg} / \mathrm{kg}$ limit. Nevertheless, Haglund et al. and unpublished data from our group suggest that doses approaching $2.0 \mathrm{mg} / \mathrm{kg}$ can still localize HGGs, meningiomas, and metastases with accuracy intraoperatively ${ }^{4}$ and could also be used to predict GTR on postoperative MRI for HGGs and metastases.

In addition to SWIG, ICG can also be used for its more conventional role as an angiography agent in brain tumor surgeries. Kim et al. showed that ICG can identify small peritumoral blood vessels in patients with intracranial meningiomas. ${ }^{25}$ Its advantage over the standard microvascular Doppler sonography is an ability to image vessels with less blood flow or smaller diameter. Similarly, ICG video angiography has extensive utility for resection of pituitary tumors. ICG video angiography during endoscopic endonasal transsphenoidal surgery can orient surgeons to the internal carotid artery, intercavernous sinus, and cav- ernous sinus with high resolution. ${ }^{26}$ Monitoring of these vessels and perforators may contribute to preservation of optic nerve and pituitary function. ICG video angiography also distinguished pituitary adenomas from normal pituitary through differential timelines for peak fluorescence intensities. For example, nonfunctioning pituitary adenomas were best visualized 7 minutes postinjection, whereas normal pituitary was best visualized 15 minutes postinjection and onward. ${ }^{27}$ Finally, as a highly vascularized tumor, hemangioblastoma can be directly visualized by ICG when used in an angiographic capacity. Preresection ICG video angiography allowed for visualization of hemangioblastoma in all patients (8/8), whereas postresection video angiography confirmed complete resection in all patients $(10 / 10) \cdot{ }^{28}$

Furthermore, because 5-ALA is a more established agent in the field of FGS, Cho et al. ${ }^{29}$ performed coadministration of 5-ALA and ICG in 4 patients with HGGs to benchmark the comparative performance of ICG. The two agents were found to have $90 \%$ concordant distributions and similar diagnostic accuracies, whereas tumor-associated necrosis demonstrated the most distinct difference, 


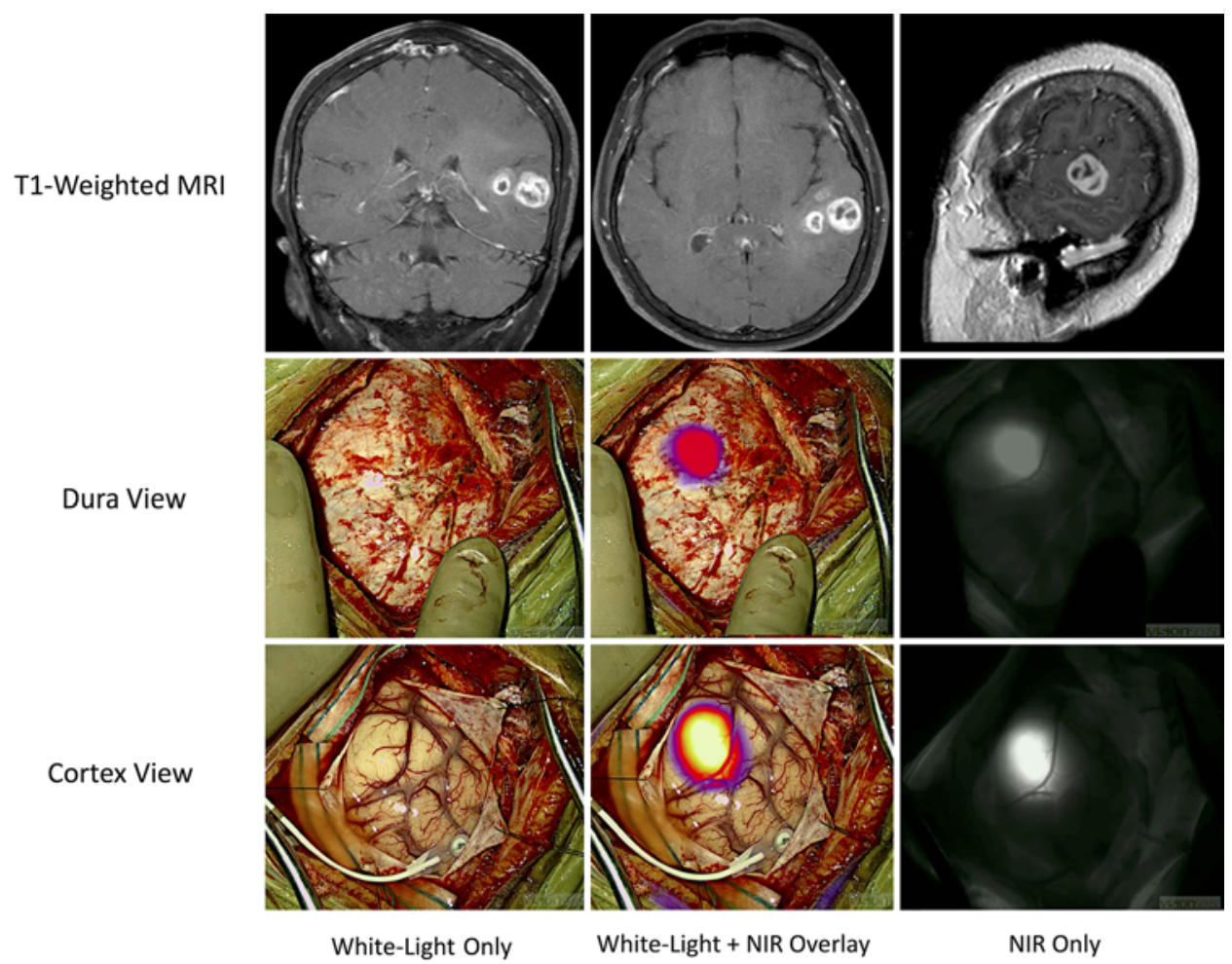

FIG. 2. FGS performed using SWIG. Demonstration of SWIG in a patient with a Gd contrast-enhancing GBM on preoperative T1weighted MRI. Transdural visualization is achieved, assisting neurosurgeons to better plan tumor access. The GBM demonstrates strong NIR fluorescence in the operating room after durotomy.

showing weak or no protoporphyrin-IX fluorescence but strong SWIG fluorescence. ${ }^{29}$ Another comparison study demonstrated that ICG was superior to 5-ALA in visualizing areas of increased angiogenesis, a process essential for metastasis of solid tumors. ${ }^{30}$

Finally, ICG has applications in tumor surgeries when not used to directly localize the tumor. Because it lacks a $\mathrm{BBB}$, the normal pituitary stalk has been observed to uptake ICG and fluoresce, allowing identification and preservation of the structure in complex skull base surgeries. ${ }^{31}$

\section{ICG in the NIR-II Window}

One of the most promising advances in optical imaging research is the discovery of the SWIR/NIR-II window, broadly defined as wavelengths in the range of 1000-2000 $\mathrm{nm}$. Whereas fluorophores in the NIR region (i.e., NIRI; 700-900 nm) generally demonstrate favorable SBR and penetration depth when compared with visible-light probes $(400-700 \mathrm{~nm})$, the SWIR window is characterized by an up to 1000 -fold reduction in photon scattering and the potential to offer micron-scale imaging resolution and centimeters of penetration depth, making it a powerful technique for the prospect of image-guided surgery. ${ }^{32}$

Until recently, SWIR fluorophores had consisted entirely of semiconducting inorganic nanomaterials (e.g., single-walled carbon nanotubes, quantum dots, and rare earth-doped nanoparticles). ${ }^{33,34}$ Despite respectable results in preclinical studies, these agents raise safety concerns due to retention in the liver and/or spleen and potential immunogenic responses, and hence are subjected to considerable regulatory hurdles prior to clinical translation. In 2018, Carr et al..$^{35}$ first demonstrated that ICG, with an emission spectra peak in NIR-I, has an off-peak > 1000$\mathrm{nm}$ tail emission. Specifically, high-contrast mesoscopic imaging of the brain vasculature was demonstrated in mice through intact skin by using ICG $(0.2 \mathrm{mg} / \mathrm{kg})$ in the NIR-II window (excitation $808 \mathrm{~nm}$, emission 1300-1620 $\mathrm{nm}){ }^{35}$ Starosolski et al. had made a similar observation independently both in vitro and in vivo. ${ }^{36}$ Subsequently, $\mathrm{Hu}$ et al. reported the first-in-human application of NIR-II imaging using ICG in 23 patients undergoing resection of hepatic neoplasms. Compared with NIR-I imaging, NIRII imaging exhibited higher tumor detection sensitivity (100\% vs 90.6\%) and SBR (5.33 vs 1.45$).{ }^{37}$ To date, no clinical trials have been conducted on NIR-II imaging using ICG in neurosurgery.

It has been acknowledged that ICG offers an unprecedented opportunity for the clinical translation of NIR-II imaging technology. ${ }^{32,38}$ However, its major limitations include relatively low photon utilization because NIR-II is only the tail portion of the emission spectrum, as well as the nonspecific targeting mechanism of the EPR effect; ${ }^{39}$ hence, researchers continue to identify strategies to improve characteristics of NIR-II imaging probes, such as synthesizing organic small molecules with a rapid excretion profile and higher imaging quality compared with free ICG ${ }^{40}$ and conjugating ICG to antibodies targeting overexpressed receptors on cancer cells. ${ }^{41}$ 


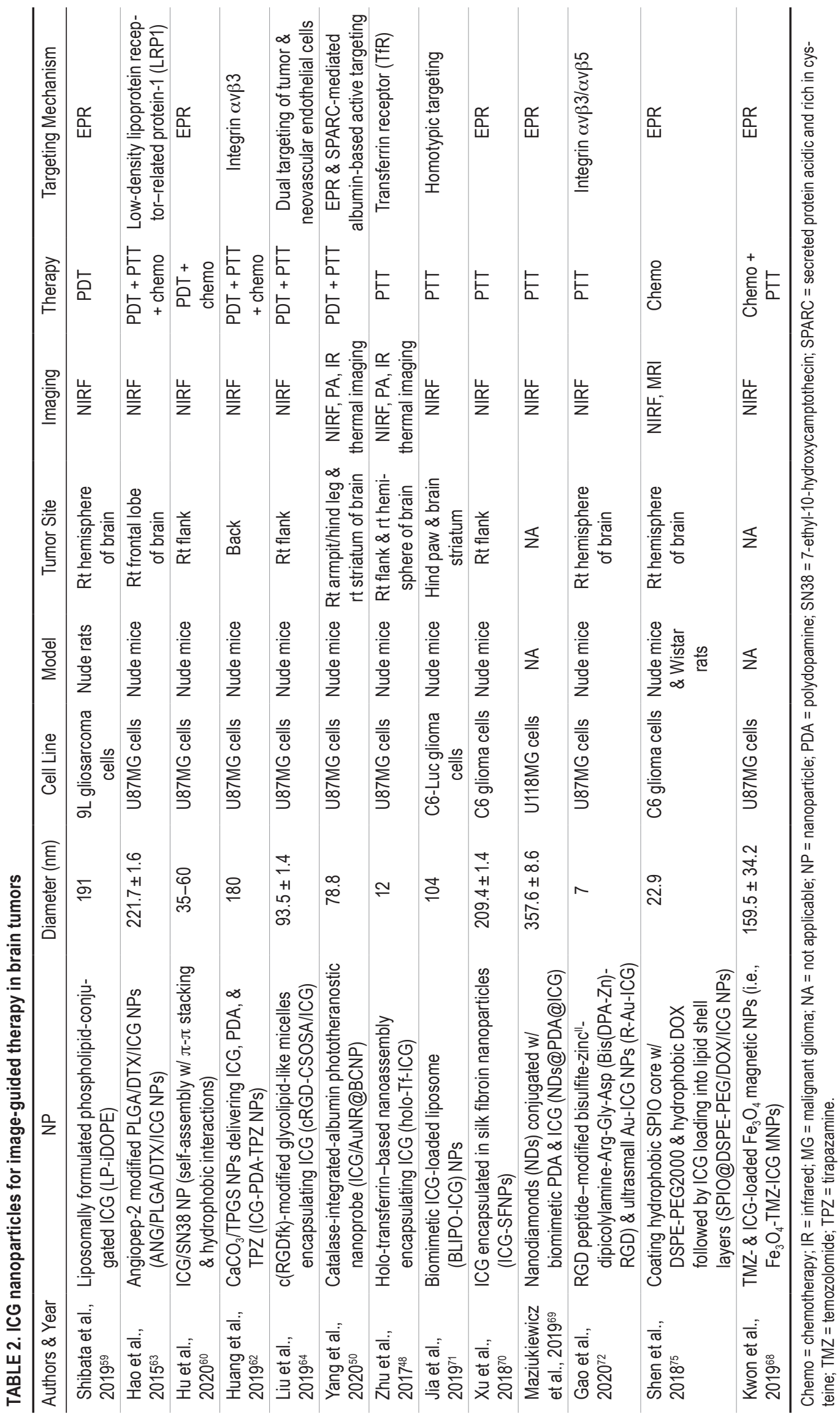


In neurosurgery, Byrd et al. recently reported the first NIR-II ICG angiography study in a large animal, demonstrating higher spatial and contrast resolutions throughout the dynamic sequence of the pig intracranial vasculature, particularly in the smallest vessels. ${ }^{42}$ The application of NIR-II imaging in brain tumor surgery has been explored in preclinical studies. Namely, Kurbegovic et al. performed FGS on orthotopic glioblastoma (GBM)-bearing nude mice by using a targeted NIR-II fluorescent probe consisting of an in-house synthesized NIR-II fluorophore (CH1055) and a uPAR-targeting peptide (AE105). ${ }^{43} \mathrm{Al}-$ though NIR-II imaging with free ICG applied in brain tumor surgery has not been reported in either preclinical or clinical studies, it may represent an important forthcoming opportunity in image-guided neurosurgery.

\section{ICG for Photoacoustic Imaging}

Photoacoustic imaging (PAI) for use in image-guided surgery has recently been investigated as a means to improve intraoperative visualization and resection of brain tumors. Also known as optoacoustic imaging, PAI is a hybrid imaging modality that combines the strong contrast of optical imaging with the high spatial resolution of ultrasound. ${ }^{44}$ The principle underlying PAI-the photoacoustic effect-occurs when molecules interact with photons. Optical absorption results in thermoelastic expansion, creating pressure perturbations that propagate as ultrasound waves. These waves are detected by an array of ultrasound transducers, which then construct an image that represents the initial photoacoustic pressure distribution based on the ultrasound waves' amplitudes and arrival times..$^{45}$ PAI systems for use in image-guided surgery consist of a source of nonionizing light, usually in the form of a pulsating laser, and an array of ultrasound transducers. ${ }^{44}$

PAI has the capability to provide higher resolution at increased penetration depths when compared with NIR fluorescence imaging, which is due to the decreased scattering of ultrasound waves compared with optical signals. ${ }^{44}$ Importantly, PAI can use exogenous dyes such as ICG, leveraging the decreased background signal inherent to NIR dyes to provide high tumor contrast. In studies in which brain and nonbrain tumors have been imaged, ICG has been combined with other contrast agents, such as gold nanoparticles, due to their superior PAI absorbance, which is orders of magnitude higher than that of ICG and other NIR dyes. ${ }^{46}$

Regardless of these findings, ICG both as the sole PAI contrast dye and in combination with other agents has been demonstrated to successfully image brain tumors in animal studies. In 2017, Thawani et al. constructed a dualmodality imaging agent using ICG-coated superparamagnetic iron oxide nanoparticle (SPION) clusters, which were used for in vivo tumor imaging in a murine flank GBM model. ${ }^{47}$ The SPIONs enabled preoperative MRI detection, whereas the ICG enabled intraoperative PAI guidance 24 hours after tail-vein injection. This study also demonstrated increased progression-free survival of mice undergoing PAI-guided surgery compared with microscopic surgery. Also in 2017, Zhu et al. demonstrated the ability of another dual-modality agent, this time for fluorescent and photo- acoustic imaging, to image orthotopic glioma models. ${ }^{48} \mathrm{At}$ 24 hours postinjection of the imaging agent, PAI of ICGholo-transferrin nanoassemblies achieved high spatial resolution and a PAI signal in the tumor region 10-fold higher than that at the preinjection time point. In 2018, Liu et al. demonstrated the ability of ICG to improve the imaging depth of an existing PAI contrast agent in an orthotopic brain glioma. ${ }^{49}$ Molybdenum disulfide nanosheets loaded with ICG achieved a $3.5-\mathrm{mm}$ imaging depth at $800 \mathrm{~nm}$, which was 2-fold deeper than with molybdenum disulfide alone. Most recently, in 2020, Yang et al. demonstrated an SBR of 6.6 at $3.1 \mathrm{~mm}$ of depth 4 hours postinjection using a biomimetic catalase-integrated-albumin phototheranostic nanoprobe with simultaneous encapsulation of ICG and gold nanoparticles. ${ }^{50}$

Therefore, ICG has been successfully used for PAI of brain tumors in animal models. Its ability to increase resolution and depth of penetration over optical imaging make it a promising imaging modality.

\section{ICG Nanoparticles: Transition to Theranostics}

Theranostics combines diagnostic imaging and therapy. Much of the development in this emerging field is powered by the recent advances in nanotechnology, which have enabled flexible synthesis of multimodal nanoparticles. Lately, the favorable imaging/biological characteristics of ICG as an NIR fluorophore have garnered interest in the incorporation of ICG into nanoparticles for the development of cancer theranostic tools. Theranostic nanomedicine is still in its infancy, primarily confined to academic settings and with few early-stage clinical studies. ${ }^{51}$ In this section, we review the synthesis and application of ICG nanoparticles in brain tumors and summarize several major therapeutic modalities that have been explored (Table 2).

\section{Photodynamic Therapy}

Photodynamic therapy (PDT) uses a photosensitizer, which upon excitation by light produces reactive oxygen species that are cytotoxic to selected cells. Several photosensitizers have been approved by the FDA; these include porfimer sodium (Photofrin) for esophageal cancer and lung cancer and 5-ALA for actinic keratosis. ${ }^{52}$ PDT research in the brain has mainly focused on gliomas. A number of early-phase clinical trials have been conducted using 5-ALA or Photofrin as photosensitizers. ${ }^{53,54}$ Notably, a phase III trial in 2018 demonstrated that FGS complemented by repetitive PDT with 5-ALA and Photofrin led to survival benefits in patients with GBM, compared with surgery and radiotherapy. ${ }^{55}$

The question of whether ICG is a good photosensitizer for PDT remains controversial. In preclinical/early clinical studies for a variety of diseases in which PDT with ICG was investigated, some found that ICG is active in PDT, whereas others claimed that it is not particularly effective. ${ }^{56}$ A study by Keller et al. found no significant temperature elevation or brain damage on histological investigation following 1-hour NIR light exposure after ICG injection in rats. ${ }^{57} \mathrm{In}$ an in vitro study, the singlet oxygen quantum yield of ICG is fairly low $(\Phi 25=0.00949)$ and 
decreases with increased concentrations, probably due to the aggregation problems of ICG at high concentrations. ${ }^{58}$

However, modifications of the dye can improve its characteristics, and ICG has been incorporated in a number of nanoparticle formulations as a fluorescent and PDT agent. For gliomas specifically, Shibata et al. developed a liposomally formulated phospholipid-conjugated ICG (LP-iDOPE) nanoparticle and found significant tumor growth suppression after using NIR irradiation in an orthotopic rat GBM model. ${ }^{59} \mathrm{Hu}$ et al. aimed to combine chemo- and phototherapy with an ICG/SN38 nanoformulation synthesized through self-assembly via hydrophobic interactions and demonstrated higher fluorescent intensity compared with free ICG, as well as enhanced antitumor activity of the dual therapy in a U87MG nude mouse flank tumor model. ${ }^{60}$ Liu et al. targeted neovascular endothelial cells in addition to tumor cells by using encapsulatedICG c(RGDfk)-modified glycolipid-like micelles (cRGDCSOSA/ICG) for dual PDT/photothermal therapy (PTT). ${ }^{61}$ Several recent studies have opted for an all-in-one cocktail approach by designing complex nanoparticles that enable imaging and therapy by using multiple modalities. ${ }^{50,62,63}$

\section{Photothermal Therapy}

PTT is a fairly new cancer treatment that uses the heat generated from the absorbed optical energy by photothermal transforming agents to achieve controlled thermal damage in tumor tissue. Whereas PDT is oxygen dependent for the generation of reactive oxygen species, PTT does not rely on oxygen and can be effective in ablating hypoxic tumors. ${ }^{64}$ Sensitizing agents under investigation include NIR light-triggered inorganic materials (transition metals, sulfide, gold, and platinum nanoparticles) ${ }^{65}$ as well as small organic molecules (cyanines, porphyrins, and polymers) ${ }^{66}$ Phase I/II trials of PTT have been conducted since 2011 for prostate cancer treatment. ${ }^{67}$

ICG is an effective NIR-absorbing PTT agent with excellent light-to-heat conversion efficiency, and numerous groups have incorporated ICG into nanoparticle formulations to enable PTT. ${ }^{68,69}$ In glioma research, Xu et al. demonstrated that ICG encapsulated in silk fibroin nanoparticles exhibited a more stable photothermal effect compared with free ICG on NIR irradiation. ${ }^{70}$ Jia et al. developed a biomimetic ICG-loaded liposome (BLIPO-ICG) nanoparticle with embedded glioma cell membrane proteins and demonstrated strong NIR fluorescence 12 hours postinjection, proof-of-concept image-guided resection, and 94.2\% tumor growth inhibition by photothermal effect. ${ }^{71}$ Recently, Gao et al. designed a two-step system in which Bis(DPA-Zn)-RGD is administered first as a prodrug, followed by administration of Au-ICG nanoparticles as a secondary agent, yielding an ultrasmall R-Au-ICG nanocluster in vivo (approximately $7 \mathrm{~nm}$ ) that can efficiently cross the BBB to enable enhanced fluorescence, PAI, and PTT. ${ }^{72}$

\section{Chemotherapy}

Traditional chemotherapy in brain tumors is limited due to several factors, including genotypic heterogeneity, high drug resistance, and, most significantly, the low BBB permeability of most chemotherapy agents. ${ }^{73}$ Polymeric controlled release and convection-enhanced deliv- ery have been two main approaches to bypass the BBB. The recent development of so-called BBB-crossing nanotechnology offers a new opportunity to integrate this conventional therapy with other emerging therapies on a nanoplatform designed to target the $\mathrm{BBB} .{ }^{74}$ Besides the multifunctional nanoparticles incorporating a chemotherapeutic moiety mentioned in the above studies, Shen et al. coated hydrophobic SPIO core with DSPE-PEG2000 and hydrophobic doxorubicin (DOX), followed by ICG loading into lipid shell layers surrounding the surface, to yield SPIO@DSPE-PEG/DOX/ICG nanoparticles with NIR fluorescence and MRI capabilities, along with the chemotherapeutic efficacy to glioma provided by DOX. ${ }^{75}$ Hence, by packaging traditional chemotherapy drugs in novel nanoparticle vehicles, the dual benefit of enhancing drug delivery and adding synergy with imaging agents like ICG can be expected.

In general, ICG has been a popular agent to be included in nanoparticle formulations as a versatile molecule conferring the theoretical basis for allowing NIR fluorescence imaging, PAI, PDT, and PTT. While other properties of ICG have been investigated in various degrees, its capability for NIR fluorescence imaging remains the most common reason to incorporate ICG in novel nanoagents. Another advantage provided by nanoparticles is the opportunity for designing active cellular targeting by using affinity ligands to bind tumor cells without accumulating in normal tissue, in contrast to free ICG, which targets neoplastic tissue through the nonspecific EPR property. This area of research would become essential for improving the precision of imaging as well as offering safe and effective application of phototherapy using ICG. With the rapid growth of nanomedicine and synthesis of a plethora of nanoparticles, one issue that comes up is the lack of a uniform metric to compare their efficacies. And as previously stated, the main challenge to bringing these new theranostic technologies to patient care remains the demonstration of safety and the regulatory approvals required prior to clinical translation. Because ICG has already been approved by the FDA for human use and its feasibility to be loaded on nanoparticle vehicles has been well documented, it presents as a compelling choice for future research on nanotherapy as the field marches closer toward translation to clinical application.

\section{Conclusions}

ICG is a safe, cheap, FDA-approved NIR fluorescent contrast agent that has been proven useful for intraoperative visualization of the most common brain tumors. Clinical results with SWIG thus far are encouraging, yet large-scale randomized trials are still required to prove its outcome benefits in patients with brain tumors. The recent discovery of ICG's tail emission in the SWIR window has opened up exciting possibilities for ICG as an NIR-II fluorophore; yet, clinical studies have not been reported for this application in intracranial tumors. Additionally, ICG, both in its free form and in combination with other agents, has been successfully used for PAI of brain tumors in animal models. The phototherapeutic properties of ICG have also been discovered and exploited mainly in the form of 
ICG nanoparticles to improve circulation time and enable active tumor targeting. Although these emerging technologies are still in the preclinical stage and may require time before reaching the bedside, they continue to expand the possibility of ICG beyond its role as a simple NIR-I optical fluorophore and to offer novel solutions to neuro-oncology patients.

\section{Acknowledgments}

This work was supported in part by the Institute for Translational Medicine and Therapeutics of the Perelman School of Medicine at the University of Pennsylvania (Dr. Lee). In addition, research reported in this publication was supported by the National Center for Advancing Translational Sciences of the NIH under award number UL1TR000003 (Dr. Lee). The content is solely the responsibility of the authors and does not necessarily represent the official views of the NIH.

\section{References}

1. Yannuzzi LA. Indocyanine green angiography: a perspective on use in the clinical setting. Am J Ophthalmol. 2011;151(5): 745-751.e1.

2. Lau CT, Au DM, Wong KKY. Application of indocyanine green in pediatric surgery. Pediatr Surg Int. 2019;35(10): 1035-1041.

3. Raabe A, Beck J, Gerlach R, et al. Near-infrared indocyanine green video angiography: a new method for intraoperative assessment of vascular flow. Neurosurgery. 2003;52(1):132-139.

4. Haglund MM, Berger MS, Hochman DW. Enhanced optical imaging of human gliomas and tumor margins. Neurosurgery. 1996;38(2):308-317.

5. Stummer W, Pichlmeier U, Meinel T, et al. Fluorescenceguided surgery with 5-aminolevulinic acid for resection of malignant glioma: a randomised controlled multicentre phase III trial. Lancet Oncol. 2006;7(5):392-401.

6. Madajewski B, Judy BF, Mouchli A, et al. Intraoperative near-infrared imaging of surgical wounds after tumor resections can detect residual disease. Clin Cancer Res. 2012; 18(20):5741-5751.

7. Lee JYK, Thawani JP, Pierce J, et al. Intraoperative nearinfrared optical imaging can localize gadolinium-enhancing gliomas during surgery. Neurosurgery. 2016;79(6):856-871.

8. Ferroli P, Acerbi F, Albanese E, et al. Application of intraoperative indocyanine green angiography for CNS tumors: results on the first 100 cases. Acta Neurochir Suppl. 2011;109: 251-257.

9. Zeh R, Sheikh S, Xia L, et al. The second window ICG technique demonstrates a broad plateau period for near infrared fluorescence tumor contrast in glioblastoma. PLoS One. 2017; 12(7):e0182034.

10. Sandow N, Klene W, Elbelt U, et al. Intraoperative indocyanine green videoangiography for identification of pituitary adenomas using a microscopic transsphenoidal approach. Pituitary. 2015;18(5):613-620.

11. Baker KJ. Binding of sulfobromophthalein (BSP) sodium and indocyanine green (ICG) by plasma alpha-1 lipoproteins. Proc Soc Exp Biol Med. 1966;122(4):957-963.

12. Cherrick GR, Stein SW, Leevy CM, Davidson CS. Indocyanine green: observations on its physical properties, plasma decay, and hepatic extraction. J Clin Invest. 1960;39(4): 592-600.

13. Stepp H, Stummer W. 5-ALA in the management of malignant glioma. Lasers Surg Med. 2018;50(5):399-419.

14. Cho SS, Salinas R, Lee JYK. Indocyanine-green for fluorescence-guided surgery of brain tumors: evidence, techniques, and practical experience. Front Surg. 2019;6:11.
15. DSouza AV. Lin H, Henderson ER, Samkoe KS, Pogue BW. Review of fluorescence guided surgery systems: identification of key performance capabilities beyond indocyanine green imaging. J Biomed Opt. 2016;21(8):080901.

16. Jeon JW, Cho SS, Nag S, et al. Near-infrared optical contrast of skull base tumors during endoscopic endonasal surgery. Oper Neurosurg (Hagerstown). 2019;17(1):32-42.

17. Ergin A, Wang M, Zhang JY, et al. The feasibility of real-time in vivo optical detection of blood-brain barrier disruption with indocyanine green. J Neurooncol. 2012;106(3):551-560.

18. Lee JYK, Pierce JT, Zeh R, et al. Intraoperative near-infrared optical contrast can localize brain metastases. World Neurosurg. 2017;106:120-130.

19. Lee JYK, Pierce JT, Thawani JP, et al. Near-infrared fluorescent image-guided surgery for intracranial meningioma. $J$ Neurosurg. 2018;128(2):380-390.

20. Cho SS, Jeon J, Buch L, et al. Intraoperative near-infrared imaging with receptor-specific versus passive delivery of fluorescent agents in pituitary adenomas. J Neurosurg. 2018; 131(6):1974-1984.

21. Cho SS, Zeh R, Pierce JT, et al. Comparison of near-infrared imaging camera systems for intracranial tumor detection. Mol Imaging Biol. 2018;20(2):213-220.

22. Cho A, Cho SS, Buch VP, et al. Second window indocyanine green (SWIG) near infrared fluorescent transventricular biopsy of pineal tumor. World Neurosurg. 2020;134:196-200.

23. Hitti FL, Lee JYK. Endoscopic resection of an intraventricular tumor with second window indocyanine green: 2-dimensional operative video. Oper Neurosurg (Hagerstown). 2018; 15(5):E53-E54.

24. Cho SS, Salinas R, De Ravin E, et al. Near-infrared imaging with second-window indocyanine green in newly diagnosed high-grade gliomas predicts gadolinium enhancement on postoperative magnetic resonance imaging. Mol Imaging Biol. 2020;22(5):1427-1437.

25. Kim J-H, Moon K-S, Jung J-H, et al. Importance of collateral venous circulation on indocyanine green videoangiography in intracranial meningioma resection: direct evidence for venous compression theory in peritumoral edema formation. J Neurosurg. 2019;132(6):1715-1723.

26. Hide T, Yano S, Shinojima N, Kuratsu J. Usefulness of the indocyanine green fluorescence endoscope in endonasal transsphenoidal surgery. J Neurosurg. 2015;122(5):1185-1192.

27. Amano K, Aihara Y, Tsuzuki S, et al. Application of indocyanine green fluorescence endoscopic system in transsphenoidal surgery for pituitary tumors. Acta Neurochir (Wien). 2019;161(4):695-706.

28. Hojo M, Arakawa Y, Funaki T, et al. Usefulness of tumor blood flow imaging by intraoperative indocyanine green videoangiography in hemangioblastoma surgery. World Neurosurg. 2014;82(3-4):e495-e501.

29. Cho SS, Sheikh S, Teng CW, et al. Evaluation of diagnostic accuracy following the coadministration of delta-aminolevulinic acid and second window indocyanine green in rodent and human glioblastomas. Mol Imaging Biol. 2020;22(5): 1266-1279.

30. Bielenberg DR, Zetter BR. The contribution of angiogenesis to the process of metastasis. Cancer J. 2015;21(4):267-273.

31. Cho SS, Buch VP, Teng CW, et al. Near-infrared fluorescence with second-window indocyanine green as an adjunct to localize the pituitary stalk during skull base surgery. World Neurosurg. 2020;136:326.

32. He S, Song J, Qu J, Cheng Z. Crucial breakthrough of second near-infrared biological window fluorophores: design and synthesis toward multimodal imaging and theranostics. Chem Soc Rev. 2018;47(12):4258-4278.

33. Jain A, Homayoun A, Bannister CW, Yum K. Single-walled carbon nanotubes as near-infrared optical biosensors for life sciences and biomedicine. Biotechnol J. 2015;10(3):447-459. 
34. McHugh KJ, Jing L, Behrens AM, et al. Biocompatible semiconductor quantum dots as cancer imaging agents. Adv Mater. 2018;30(18):e1706356.

35. Carr JA, Franke D, Caram JR, et al. Shortwave infrared fluorescence imaging with the clinically approved near-infrared dye indocyanine green. Proc Natl Acad Sci U S A. 2018; 115(17):4465-4470.

36. Starosolski Z, Bhavane R, Ghaghada KB, et al. Indocyanine green fluorescence in second near-infrared (NIR-II) window. PLoS One. 2017;12(11):e0187563.

37. Hu Z, Fang C, Li B, et al. First-in-human liver-tumour surgery guided by multispectral fluorescence imaging in the visible and near-infrared-I/II windows. Nat Biomed Eng. 2020; 4(3):259-271.

38. Zhu S, Yung BC, Chandra S, et al. Near-infrared-II (NIR-II) bioimaging via off-peak NIR-I fluorescence emission. Theranostics. 2018;8(15):4141-4151.

39. Li J-B, Liu H-W, Fu T, et al. Recent progress in small-molecule near-IR probes for bioimaging. Trends Chem. 2019;1(2): 224-234.

40. Antaris AL, Chen H, Cheng K, et al. A small-molecule dye for NIR-II imaging. Nat Mater. 2016;15(2):235-242.

41. Tsuboi S, Jin T. Shortwave-infrared (SWIR) fluorescence molecular imaging using indocyanine green-antibody conjugates for the optical diagnostics of cancerous tumours. RSC Adv. 2020;10(47):28171-28179.

42. Byrd BK, Marois M, Tichauer KM, et al. First experience imaging short-wave infrared fluorescence in a large animal: indocyanine green angiography of a pig brain. J Biomed Opt 2019;24(8):080501.

43. Kurbegovic S, Juhl K, Chen H, et al. Molecular targeted NIRII probe for image-guided brain tumor surgery. Bioconjug Chem. 2018;29(11):3833-3840.

44. Moore C, Jokerst JV. Strategies for image-guided therapy, surgery, and drug delivery using photoacoustic imaging. Theranostics. 2019;9(6):1550-1571.

45. Mallidi S, Luke GP, Emelianov S. Photoacoustic imaging in cancer detection, diagnosis, and treatment guidance. Trends Biotechnol. 2011;29(5):213-221.

46. Li W, Chen X. Gold nanoparticles for photoacoustic imaging. Nanomedicine (Lond). 2015;10(2):299-320.

47. Thawani JP, Amirshaghaghi A, Yan L, et al. Photoacousticguided surgery with indocyanine green-coated superparamagnetic iron oxide nanoparticle clusters. Small. 2017;13(37).

48. Zhu M, Sheng Z, Jia Y, et al. Indocyanine green-holo-transferrin nanoassemblies for tumor-targeted dual-modal imaging and photothermal therapy of glioma. ACS Appl Mater Interfaces. 2017;9(45):39249-39258.

49. Liu C, Chen J, Zhu Y, et al. Highly sensitive $\mathrm{MoS}_{2}$-indocyanine green hybrid for photoacoustic imaging of orthotopic brain glioma at deep site. Nanomicro Lett. 2018;10(3):48.

50. Yang Z, Du Y, Sun Q, et al. Albumin-based nanotheranostic probe with hypoxia alleviating potentiates synchronous multimodal imaging and phototherapy for glioma. ACS Nano. 2020;14(5):6191-6212.

51. Singh D, Dilnawaz F, Sahoo SK. Challenges of moving theranostic nanomedicine into the clinic. Nanomedicine (Lond). 2020;15(2):111-114.

52. Mahmoudi K, Garvey KL, Bouras A, et al. 5-aminolevulinic acid photodynamic therapy for the treatment of high-grade gliomas. J Neurooncol. 2019;141(3):595-607.

53. Muller PJ, Wilson BC. Photodynamic therapy of brain tumors-a work in progress. Lasers Surg Med. 2006;38(5): 384-389.

54. Aziz F, Telara S, Moseley H, et al. Photodynamic therapy adjuvant to surgery in metastatic carcinoma in brain. Photodiagn Photodyn Ther. 2009;6(3-4):227-230.

55. Eljamel MS, Goodman C, Moseley H. ALA and Photofrin fluorescence-guided resection and repetitive PDT in glio- blastoma multiforme: a single centre Phase III randomised controlled trial. Lasers Med Sci. 2008;23(4):361-367.

56. Giraudeau C, Moussaron A, Stallivieri A, et al. Indocyanine green: photosensitizer or chromophore? Still a debate. Curr Med Chem. 2014;21(16):1871-1897.

57. Keller E, Ishihara H, Nadler A, et al. Evaluation of brain toxicity following near infrared light exposure after indocyanine green dye injection. J Neurosci Methods. 2002; 117(1):23-31.

58. Ruhi MK, Ak A, Gülsoy M. Dose-dependent photochemical/ photothermal toxicity of indocyanine green-based therapy on three different cancer cell lines. Photodiagn Photodyn Ther. 2018;21:334-343.

59. Shibata S, Shinozaki N, Suganami A, et al. Photo-immune therapy with liposomally formulated phospholipid-conjugated indocyanine green induces specific antitumor responses with heat shock protein-70 expression in a glioblastoma model. Oncotarget. 2019;10(2):175-183.

60. Hu S, Dong C, Wang J, et al. Assemblies of indocyanine green and chemotherapeutic drug to cure established tumors by synergistic chemo-photo therapy. J Control Release. 2020; 324:250-259.

61. Liu Y, Dai S, Wen L, et al. Enhancing drug delivery for overcoming angiogenesis and improving the phototherapy efficacy of glioblastoma by ICG-loaded glycolipid-like micelles. Int J Nanomedicine. 2020;15:2717-2732.

62. Huang X, Wu J, He M, et al. Combined cancer chemophotodynamic and photothermal therapy based on ICG/ PDA/TPZ-loaded nanoparticles. Mol Pharm. 2019;16(5): 2172-2183.

63. Hao Y, Wang L, Zhao Y, et al. Targeted imaging and chemophototherapy of brain cancer by a multifunctional drug delivery system. Macromol Biosci. 2015;15(11):1571-1585.

64. Liu Y, Bhattarai P, Dai Z, Chen X. Photothermal therapy and photoacoustic imaging via nanotheranostics in fighting cancer. Chem Soc Rev. 2019;48(7):2053-2108.

65. Day ES, Morton JG, West JL. Nanoparticles for thermal cancer therapy. J Biomech Eng. 2009;131(7):074001.

66. Jung HS, Verwilst P, Sharma A, et al. Organic moleculebased photothermal agents: an expanding photothermal therapy universe. Chem Soc Rev. 2018;47(7):2280-2297.

67. Rastinehad AR, Anastos H, Wajswol E, et al. Gold nanoshelllocalized photothermal ablation of prostate tumors in a clinical pilot device study. Proc Natl Acad Sci U S A. 2019; 116(37):18590-18596.

68. Kwon YM, Je J-Y, Cha SH, et al. Synergistic combination of chemo-phototherapy based on temozolomide/ICG-loaded iron oxide nanoparticles for brain cancer treatment. Oncol Rep. 2019;42(5):1709-1724.

69. Maziukiewicz D, Grześkowiak BF, Coy E, et al. NDs@ PDA@ICG conjugates for photothermal therapy of glioblastoma multiforme. Biomimetics (Basel). 2019;4(1):3.

70. Xu H-L, ZhuGe DL, Chen PP, et al. Silk fibroin nanoparticles dyeing indocyanine green for imaging-guided photo-thermal therapy of glioblastoma. Drug Deliv. 2018;25(1):364-375.

71. Jia Y, Wang X, Hu D, et al. Phototheranostics: active targeting of orthotopic glioma using biomimetic proteolipid nanoparticles. ACS Nano. 2019;13(1):386-398.

72. Gao H, Chu C, Cheng Y, et al. In Situ formation of nanotheranostics to overcome the blood-brain barrier and enhance treatment of orthotopic glioma. ACS Appl Mater Interfaces. 2020;12(24):26880-26892.

73. Tang W, Fan W, Lau J, et al. Emerging blood-brain-barriercrossing nanotechnology for brain cancer theranostics. Chem Soc Rev. 2019;48(11):2967-3014.

74. Belykh E, Shaffer KV, Lin C, et al. Blood-brain barrier, blood-brain tumor barrier, and fluorescence-guided neurosurgical oncology: delivering optical labels to brain tumors. Front Oncol. 2020;10:739. 
75. Shen C, Wang X, Zheng Z, et al. Doxorubicin and indocyanine green loaded superparamagnetic iron oxide nanoparticles with PEGylated phospholipid coating for magnetic resonance with fluorescence imaging and chemotherapy of glioma. Int J Nanomedicine. 2018;14:101-117.

76. Teng CW, Cho SS, Singh Y, et al. Second window ICG predicts gross-total resection and progression-free survival during brain metastasis surgery. J Neurosurg. In press.

\section{Disclosures}

The authors report no conflict of interest concerning the materials or methods used in this study or the findings specified in this paper.

\section{Author Contributions}

Conception and design: Teng. Acquisition of data: Teng. Analysis and interpretation of data: Teng, Huang, Arguelles, Zhou. Drafting the article: Teng, Huang, Arguelles, Zhou. Critically revising the article: Teng, Huang, Arguelles, Zhou. Reviewed submitted version of manuscript: all authors. Approved the final version of the manuscript on behalf of all authors: Lee. Study supervision: Lee, Teng, Harmsen.

\section{Supplemental Information \\ Video}

Video Abstract. https://vimeo.com/488051911.

\section{Correspondence}

John Y. K. Lee: Hospital of the University of Pennsylvania, Philadelphia, PA. leejohn@pennmedicine.upenn.edu. 